\title{
Research on HOXA11-AS in Malignant Tumors
}

\author{
Juncheng Guo', Jinfang Zheng2*, Yijun Yang1 \\ ${ }^{1}$ Affiliated Haikou Hospital of Central South University Xiangya School of Medicine, Haikou, China \\ ${ }^{2}$ Liver and Gallbladder Surgery, Hainan General Hospital, Haikou, China \\ Email: *g2002m@163.com
}

How to cite this paper: Guo, J.C., Zheng, J.F. and Yang, Y.J. (2021) Research on HOXA11-AS in Malignant Tumors. Journal of Cancer Therapy, 12, 19-30. https://doi.org/10.4236/jct.2021.121003

Received: December 6, 2020

Accepted: January 11, 2021

Published: January 14, 2021

Copyright $\odot 2021$ by author(s) and Scientific Research Publishing Inc. This work is licensed under the Creative Commons Attribution International License (CC BY 4.0).

http://creativecommons.org/licenses/by/4.0/

(c) (i) Open Access

\begin{abstract}
Long-chain non-coding RNA HOXA11 antisense RNA (HOXA11-AS) is a kind of lncRNA discovered in recent years. Long-chain non-coding RNA (LncRNA) is an important regulatory factor of protein-coding genes, especially the disorder of LncRNA in more and more diseases which are found, including cancer. HOXA11-AS was first discovered in mouse embryo cDNA library using probes, and then it was discovered by scholars and played an important role in human cervical cancer, gastric cancer, glioma and other malignant tumor cells. Overexpression of HOXA11-AS has been found to promote cell proliferation, migration and tumor invasion, and has a carcinogenic effect. HOXA11AS can promote tumor proliferation, metastasis and other malignant biological behaviors by interacting with miRNA and EZH2 protein, and is considered to be carcinogenic IncRNA. The discovery of HOXA11-AS provides new ideas for tumor prevention and treatment.
\end{abstract}

\section{Keywords}

Long Non-Coding RNA, HOXA11-AS, Malignant Tumor

\section{Introduction}

In 1995, Potter et al. found that the antisense strand of HOXAll can encode multiple transcripts when studying the effect of HOXAll gene on mouse fertility function [1]. In the human genome, more than $80.4 \%$ of genes can be transcribed, and only $2 \%$ of the transcripts can be further translated into proteins. The remaining massive transcripts are called noncoding RNA (ncRNA) [2]. Long noncoding RNA (10 ng noncoding RNA, IncRNA) refers to noncoding RNA with a length of more than 200 nucleotides [3]. In the past ten years, more and more studies have shown that lncRNA has very important biological functions in tumors and non-tumor diseases, and can regulate many pathophysiological pro- 
cesses. Yuan et al. [4] found that IncRNA ATB regulates the expression of ZEB1/2 through the mechanism of competitive endogenous RNA (ceRNA) adsorption of miR-200 family in liver cancer, and promotes the epithelium of liver cancer cells. Mesenchymal transition (epithelial-mesenchymal transition, EMT) further enhances the invasion ability of liver cancer cells; and lncRNA-ATB can bind to iL-1lmRNA and induce IL-11 autocrine, activate STAT3 signaling pathway to promote the colonization of metastatic liver cancer cells. Long et al. [5] found that IncRNA Tugl is significantly low expressed in podocytes in diabetic mice, lncRNA-Tugl binds to the specific binding site upstream of the Ppargcla gene, enhances promoter activity, and promotes peroxisome proliferation without activation of receptor $r$ (PPARr). The expression of agent a (PGC-1a), thereby improving the damage of podocyte mitochondria in a high-glycemic environment, thus may alleviate the progression of diabetes.

HOXAlI-AS is a newly discovered long-chain non-coding RNA. In recent years, HOXA11-AS has received extensive attention in both oncology and non-tumor fields. In malignant tumors, a large number of studies have shown that the expression level of HOXA11-AS in tumor tissues is significantly increased, and it has the effect of promoting tumor occurrence and development: but there are also a few reports showing that HOXA11-AS exhibits low expression in some tumor tissues, which can inhibit The function of tumor development [6]. In nonneoplastic diseases, HOXA11-AS inhibits the proliferation of chondrocytes and can induce their apoptosis, leading to delayed fracture healing [7]. In addition, HOXA11-AS promotes the inflammatory response during the formation of carotid arteries, leading to the progression of diabetic carotid arteries [8]. In addition, HOXAll-AS promotes the differentiation of adipose stem cells into adipocytes and the deposition of fat in cells, thus leading to the occurrence of obesity [9]. HOXAll-AS has different biological characteristics in different diseases and different tissues, which perfectly interprets the tissue and cell specificity of IncRNA [10]. This article reviews the existing research on HOXA11-AS, and provides references for further research on the relationship between HOXA11-AS and malignant tumors.

\section{Epithelial Ovarian Cancer}

Epithelial ovarian cancer has the highest mortality rate among reproductive system tumors, and HOXAll is related to reproductive ability [11], and the expression level of HOXA11-AS in the endometrium is regulated by progesterone [12], so it is first thought of HOXAll-AS may play a special physiological function in reproductive system tumors. Richards et al. [13] believed that single nucleotide polymorphisms (SNPs) can affect HOXA11-AS and inhibit the growth of ovarian cancer; experimental studies by Kim et al. [14] confirmed that HOXA11-AS is abnormally high in cervical cancer Expressed and overexpressed HOXA11-AS can promote the proliferation and invasion and metastasis of cervical cancer tumor cells, and regulate the cell stemness and epithelial mesenchymal process 
of tumor cells. At the same time, HOXA11-AS can be used as an independent assessment of the overall survival of cervical cancer patients. Factors; found in osteosarcoma: HOXA11-AS can promote the proliferation of osteosarcoma cells through the interaction of HOXA11-AS/miR-124-3p/ROCK1 signaling axis [15] [16]. In fact, epithelial ovarian cancer is the first disease to be shown to be related to HOXA11-AS. Richards et al. [17] analyzed the genome-wide joint data of 1201 serous ovarian cancer cases and 2009 healthy controls and showed that there is a mutation $(\mathrm{A}>\mathrm{T})$ at the rsl7427875 locus of an exon in HOXAll-AS. This locus variation It is related to the risk of serous ovarian cancer; after this site is mutated from A to $\mathrm{T}$, the survival, proliferation, migration and invasion ability of ovarian cancer cells is reduced, and the tumorigenesis ability in the body is also inhibited; 18 pairs of tumors were detected, and the normal paired ovarian tissues The expression level of HOXA11-AS found that the expression of epithelial ovarian tumor tissue was significantly lower than that of normal ovarian tissue, suggesting that HOXA11-AS may have a tumor suppressor effect; and the expression level of the adjacent genes HOXA11 and HOXAl3 is the same as that of HOXA11-AS There is no obvious correlation between the level and the mutation of this site. In another study, YIM et al. [18] found that the expression of HOXA11-AS in serous ovarian cancer tissues was 77 times that of non-cancerous tissues. The high expression level of HOXA11-AS and histopathological grades and preoperative carcinoembryonic antigen CAl25 levels are significantly related: HOXA11-AS overexpression in serous ovarian cancer cells leads to increased cell proliferation, invasion and migration: In addition, HOXA11-AS is also related to the expression of genes related to cell invasion, migration and EMT; multi-factor analysis shows that, HOXA11-AS is an important prognostic factor affecting disease progression and death. Patients with higher levels of HOXA11-AS expression have significantly shorter tumor progression-free periods and overall survival. There are also reports that the two different results of the same disease may be due to different specimen methods (such as different races). Therefore, the accuracy of the two results can only be distinguished by a comprehensive comparison of the baseline levels of various indicators of the clinical specimens and more in-depth research.

\section{Non-Small Cell Lung Cancer}

Non-small cell lung cancer (NSCLC) is the most common type of lung cancer. Interestingly, HOXA11-AS also has a tumor-promoting effect on tumors in NSCLC. HOXA11-AS is significantly up-regulated in NSCLC tissues, which is 2.38 times that of normal tissues, and the level of HOXA11-AS is positively correlated with the prognosis of NSCLC patients [19]. There are currently studies on HOXAll-AS in non-small cell lung cancer, and the results show that HOXAll-AS is significantly highly expressed in non-small cell lung cancer. Zhang et al. [20] used small interfering RNA technology to interfere with the expression of HOXAll-AS in the A549 cell line of non-small cell lung cancer, and then used 
gene chips to screen the changes in transcripts, and found that 277 genes were significantly up-regulated and 80 genes The expression is significantly downregulated, and the results of enrichment analysis of GO and KEGG pathways show that HOXAll-AS may be related to DOCK8 and TGF-B pathways. Subsequently, on this basis, another study of the group reported that HOXA11-AS is highly expressed in non-small cell lung cancer tissues and cell lines. The proliferation, migration, invasion, and invasion of non-small cell lung cancer cells after HOXAll-AS knockout Both tumorigenicity and angiogenesis are inhibited, while apoptosis is increased, and the cell cycle is arrested in GO/G1 or G2/M phase [21]. Further research found that HOXA11-AS may exert its above biological functions in non-small cell lung cancer by regulating the expression of miR-642b-3p and PDE4D [22]. Chen et al. [23] found that the expression level of HOXA11-AS in non-small cell lung cancer was significantly higher than that in normal tissues adjacent to cancer, and the high expression level of HOXA11-AS was related to poor tumor TMN staging, lymph node metastasis and clinical prognosis; mechanism; On the one hand, HOXAll-AS inhibits the expression of miR-200b by promoting the binding of EZH 2 (zeste homolog 2) and DNMT1 in the miR-200b promoter region and promotes the occurrence of EMT in tumor cells. In addition, Yu et al. [24] also found that HOXA11-AS is up-regulated in non-small cell lung cancer tissues and cell lines, and is positively correlated with tumor size and lymph node metastasis; HOXA11-AS regulates the expression of SP1 through competitive adsorption of miR-124, And then promote the proliferation and invasion of tumor cells. In addition, in a recent study, Zhao et al. [25] found that HOXAll-AS increased the resistance of adenocarcinoma cells to the chemotherapy drug cisplatin by regulating the miR-454-3p/Star3 pathway. In summary, HOXA11-AS is significantly highly expressed in non-small cell lung cancer, and promotes tumor occurrence and progression, and increases the resistance of adenocarcinoma to chemotherapeutics. The higher expression level of HOXA11-AS and poor clinical prognosis Related. Therefore, HOXA11-AS has great potential value in the diagnosis and treatment of non-small cell lung cancer.

\section{Gastric Cancer}

In China, the incidence of gastric cancer ranks second among all malignant tumors, and the incidence of gastric cancer in rural areas ranks first among all malignant tumors. Gastric cancer is still prone to metastasis and spread after radical surgery and adjuvant chemotherapy, and nearly $60 \%$ of postoperative patients have recurrence or metastasis [26]. Studies have found that the expression of HOXAll-AS is significantly increased in gastric cancer. Overexpression of HOXAll-AS promotes the proliferation, migration and invasion of gastric cancer cells, inhibits cell apoptosis and blocks cell cycle [27]. Results The expression of HOXAll-As in gastric cancer tissues and gastric cancer cells was increased $(\mathrm{P}<$ $0.05)$; the nuclear and cytoplasmic separation experiment showed that the dis- 
tribution of HOXAll-As in the cytoplasm of gastric cancer cells was higher than that in the nucleus $(\mathrm{P}<0.05)$; IncRNA was overexpressed HOXAll-AS can promote the proliferation, migration and invasion of gastric cancer cells $(\mathrm{P}<0.05)$, inhibit cell apoptosis and block cell cycle. Conclusion HOXAll-As is highly expressed in gastric cancer, can promote the tumor cell behavior of gastric cancer cells, and can be used as a candidate molecular target for the treatment of gastric cancer [28]. Sun et al. [29] analyzed clinical data and found that elevated HOXA11AS expression levels are associated with shorter survival and poor prognosis; multilevel studies have shown that HOXAll-AS is triggered by the transcription factor E2F1 and acts as EZH2/ in the nucleus. The molecular scaffold of LSD1/ DNMT1 appears; on the other hand, HOXA11-AS can competitively adsorb miR-1297 and relieve its ability to inhibit the translation of EZH2 protein. On this basis, the group further studied and found that HOXAll-AS can also bind to WDR5 in gastric cancer cells to promote the expression of B-catenin, bind to EzH2 to inhibit the expression of p21, and bind to STAUl to induce KLF2mRNA in gastric cancer cells. The degradation of [30]. It can be seen that HOXAll-AS has carcinogenic effects in both the transcription and post-transcription of gastric cancer, and has the possibility of becoming a diagnostic marker and potential therapeutic target for gastric cancer.

\section{Colorectal Cancer}

We found and reported that the expression of HOXAll-AS in the primary colorectal cancer with liver metastasis was significantly increased [31]. In this part of the study, we verified by qRT-PCR and found that the expression of HOXAll-AS in the primary colorectal cancer with liver metastasis was also significant compared with the primary colorectal cancer tissue without metastasis. Increased. By knockout or overexpression HOXAll. AS can effectively inhibit or promote the migration and invasion of colon cancer cells. Colorectal cancer is another gastrointestinal tumor, and its mortality rate ranks fourth among all tumors. There are only two studies on HOXA11-AS in colorectal cancer, but the interesting thing is that the results of the two studies are not consistent. First, a study examined 84 cases of colon cancer tissues and found that the expression level of HOXAll-AS in cancer tissues was significantly lower than that in adjacent tissues, and HOXAll-AS in three colorectal cell lines (HcT8, HCTll6, and RKO) were also It is lower than that in normal colorectal cell line (CCD.18Co); analysis of clinicopathological data shows that the low expression of HOXA11-AS is closely related to the size of cancer tissue, advanced tumor metastasis stage, lymph node metastasis and carcinoembryonic antigen level [32]. In another study, Chen et al. [33] found that HOXA11-AS had lower levels in colorectal cancer with liver metastasis and highly aggressive cell lines (Col0205, HCT116, Lovo and Sw620) than colon cancer tissues without liver metastasis. The expression level of HOXA11AS in aggressive cytoplasmic strains (Caco-2 and SW480) was significantly increased. Further research found that HOXA11-AS regulates PAD12 expression 
through competitive adsorption of miR-125a-5p to promote the invasion and metastasis of colorectal cancer. The selection of tissue specimens and cell lines is quite different, so it does not mean that the results of the two are contradictory. It is also possible that the expression level of HOXA11-AS in colon cancer tissue is lower than that in normal tissues, and colorectal cancer with liver metastasis The expression level of HOXA11-AS in tissues is only higher than that of nonmetastatic tissues but not higher than that of normal cancer tissues.

\section{Glioma}

Studies have found that HOXA11-AS is closely related to the growth and metastasis of glioma. Some scholars believe that HOXA11-AS can be a marker for the classification and classification of glioma. Wang et al. [34] used gene chip detection and found that the expression of HOXAll-AS in human glioma tissue was significantly increased, which was closely related to the grade and poor prognosis of glioma; multivariate Cox regression analysis showed that HOXA11-AS was more An independent prognostic factor for glioblastoma, its expression level is related to the molecular subtype of glioma in the tumor genome map; functional experiments have shown that overexpression of HOXA11-AS promotes cell proliferation, while interference with HOXA11-AS regulates cell cycle And inhibit cell proliferation. A study showed that compared with adjacent normal brain tissues and normal human astrocyte cell lines, the expression level of HOXAll-AS in glioma tissues and cell lines was significantly increased, and the high expression level of HOXAll-AS was similar to that in glioma patients. It is related to the shortening of overall survival; knocking out HOXA11-AS can inhibit the proliferation, migration and invasion of glioma cells, and inhibit the growth of tumors in vivo; the author found that HOXA11-AS acts as ceRNA to adsorb miR-214-3p to EZH2 The expression of has a positive regulatory effect [35]. In another study, Cui et al. [36] also showed that HOXAll-AS is highly expressed in glioma tissues and cell lines. Patients with high HOXAll-AS expression levels have a shorter survival time and poor prognosis: HOXA11-AS passed Adsorb miR-140-5p to inhibit the proliferation of glioma cells, induce cell cycle arrest in G0/G1 phase and promote cell apoptosis. In this report, the authors did not further study the specific downstream target genes of miR-140-5p. In a recent study on HOXAll-AS in gliomas, Sun et al. [37] found that the expression of HOXAll-AS in glioma tissues was significantly increased, and the increased expression of HOXAll-AS was associated with poor tumor grades. It is closely related to clinical prognosis; HOXA11-AS promotes cell proliferation, invasion and migration through competitive adsorption of miR-124-3p, and inhibits cell apoptosis. This study did not further reveal the target genes of miR-124-3p. To sum up, HOXAll-AS is highly expressed in gliomas and plays a role in promoting tumors, and its high expression predicts poor clinical prognosis. Therefore, HOXAll-AS has become a diagnostic marker and potential for glioma. The possibility of treatment targets. 


\section{Liver Cancer}

In recent years, studies have found that HOXA11-AS can also promote the occurrence and development of hepatocellular carcinoma (HCC). In vitro experiments found that the expression level of HOXA11-AS in HCC tissues was significantly increased, more than double the expression in normal tissues, and the overall survival time of patients with high HOXA11-AS expression was significantly shorter than that of patients with low expression, while HOXA11-AS A decrease in AS can significantly inhibit the tumorigenic ability of HCC cells [38]. Liu et al. [39] tested 66 liver cancer tissue specimens (male, 60 female, 6) and liver cancer cell lines (HepG2, Hep3B, Bel-7402, and SMMC-7721) and found that their HOXAll-AS expression level was higher than that of adjacent tissues and normal liver The epithelial cell line (L02) is obviously high, interferes with HOXAll-AS, inhibits the proliferation of liver cancer cells, promotes apoptosis and arrests the cell cycle in G1 phase; HOXAll-AS promotes the binding of EZH2 to the DUSP5 promoter region and inhibits the transcription of DUSP5 And then promote the occurrence and progression of liver cancer. A recent item also showed that HOXAll-AS plays a role in promoting cancer in liver cancer. The team detected the expression of HOXAll-AS in liver cancer tissues (40 pairs) and cell lines (MHCC.97H-Bel-7404, SMMC7721 and QGY-7703) Compared with the adjacent tissues and normal liver epithelial cell line (HL-7702), HOXAll-AS promotes the proliferation and invasion of liver cancer and can induce EMT; further research shows that HOXAll-AS promotes cancer by adsorbing miR-214-3p effect. In another study, Yu et al. [40] found that the expression level of HOXA11-AS in liver cancer tissues (40 pairs) and cell lines (HepG2, Hep3B, and MHCC.97H) was relative to that of adjacent tissues and normal liver epithelial cell lines ( L02) is significantly higher, HOXAll-AS inhibits the proliferation of liver cancer cells, promotes apoptosis, and delays the development of the cell cycle from G1 to GO phase; in terms of pathways, HOXAll-AS inhibits the expression of LATS1 by recruiting EZH2. In the last report, there are contradictions between the conclusions of functional experiments and tumor-promoting effects. LATS 1 (Large tumor suppressor 1) is one of the core members of the hippo signaling pathway, which is responsible for regulating cell cycle and apoptosis. It is lowly expressed in a variety of tumors. Overexpression of LATS1 can inhibit the growth of tumor cells [41]. Studies have detected that LATS1 is up-regulated in HepG2 and Hep3B after interfering with HOXAll-AS. Therefore, HOXA11-AS should promote tumor cell proliferation and inhibit cell apoptosis, which contradicts the results of functional experiments in the article. Combining the first two reports and its own contradictory conclusions, the selection of materials for the three studies is not much different. Therefore, we have reason to believe that the colleagues in the latter study may have problems or experimental errors or other problems in a certain part of the functional experiment. The undiscovered regulatory effect is stronger than the LATS1 pathway, so more indepth research is needed. 


\section{Laryngeal Squamous Cell Carcinoma}

Squamous cell carcinoma of the larynx. Laryngeal squamous cell carcinoma (LSCC) is a common malignant tumor. Laryngeal cancer of the upper respiratory tract is graded, followed by the most common malignant tumors of the head and neck [42]. Qu et al. [43] used gene chip and qRT-PCR technology to study and found HOXAll. The expression level of AS in laryngeal squamous cell carcinoma tissue is significantly higher than that in adjacent tissues. HOXAll-AS is significantly expressed in the nucleus and is closely related to tumor grade, cervical lymph node metastasis and clinical stage; Kaplan. Meier analysis showed that the high expression level of HOXAll-AS indicates a poor prognosis for patients with laryngeal squamous cell carcinoma, and interference with HOXAll-AS significantly inhibits the growth, migration and invasion of laryngeal squamous cell carcinoma. Sun Xiaoyan et al. [44] found that HOXAl1-AS was up-regulated in esophageal squamous cell carcinoma tissues and cell lines, and its high expression level was related to the histological grade and lymph node metastasis of patients with esophageal squamous cell carcinoma, and was related to the patient's age, gender, and tumor invasion. Depth has nothing to do with TNM staging. The results of Cox multivariate regression analysis showed that HOXAll-AS expression is an independent risk factor affecting the median survival time and median progression-free survival time of patients with esophageal squamous cell carcinoma. Zhang Li et al. [45] found that the expression of HOXAll-AS in head and neck squamous cell carcinoma tissues and cell lines is higher than that in normal tissues and cell lines, and it can promote the migration of head and neck squamous cell carcinoma cells.

\section{Summary and Outlook}

In recent years, lncRNA has become a new hot spot in modern genetic researches. More and more studies have found that lncRNA is dysregulated in various cancer types, and the abnormal expression of lncRNAs actually participates in all stages of cancer development, included the occurrence and metastasis of cancer. At present, the understanding of HOXA11-AS has made great progress; now it is believed that HOXA11-AS can have a cancer-promoting effect on most tumors, such as glioma, gastric cancer and non-small cell lung cancer, etc., and the mechanism and target of action not exactly. However, in general, the mechanism of action of HOXA11-AS is still at an early stage, and many detailed and in-depth studies need to be continued. For example, the cancer-promoting mechanism of HOXA11-AS for laryngeal squamous cell carcinoma is not clear, HOXA11-AS and tumors Research on the relationship between chemotherapy resistance and patient response after treatment. However, with the deepening of research and the continuous improvement of research methods and technologies, the mechanism of HOXA11-AS on tumor proliferation and metastasis will eventually be explored.HOXA11-AS also has great potential to become a new target for disease prevention, diagnosis and treatment point. 


\section{Acknowledgements}

This study was supported by Hainan Provincial Basic and Applied Basic Research Program (Natural Science Field) High-level Talent Project in 2019 (No. 2019RC373).

\section{Conflicts of Interest}

The authors declare no conflicts of interest regarding the publication of this paper.

\section{References}

[1] Potter, S.S. and Branford, W.W. (1998) Evolutionary Conservation and TissueSpecific Processing of Hoxal1 Antisense Transcripts. Mammalian Genome, 9, 799 806. https://doi.org/10.1007/s003359900870

[2] Consortium, E.P. (2012) An Integrated Encyclopedia of DNA Elements in the Human Genome. Nature, 489, 57-74. https://doi.org/10.1038/nature11247

[3] Cox, A.D., Fesik, S.W., Kimmelman, A.C., Luo, J. and Der, C.J. (2014) Drugging the Undruggable RAS: Mission Possible? Nature Reviews Drug Discovery, 13, 828-851. https://doi.org/10.1038/nrd4389

[4] Yuan, J.H., Yang, F., Wang, F., Ma, J.-Z., Guo, Y.-J., Tao, Q.-F., et al. (2014) A Long Noncoding RNA Activated by TGF-Beta Promotes the Invasion-Metastasis Cascade in Hepatocellular Carcinoma. Cancer Cell, 25, 666-681. https://doi.org/10.1016/j.ccr.2014.03.010

[5] Long, J.Y., Badal, S.S., Ye, Z.C., Wang, Y., Ayanga, B.A., Galvan, D.L., Green, N.H., et al. (2016) Long Noncoding RNA Tu91 Regulates Mitochondrial Bioenergetics in Diabetic Nephropathy. Journal of Clinical Investigatio, 126, 4205-4218. https://doi.org/10.1172/JCI87927

[6] Yu, J., Hong, J.F., Kang, J., Liao, L.H. and Li, C.D. (2017) Promotion of LncRNA HOXAI 1-AS on the Proliferation of Hepatocellular Carcinoma by Regulating the Expression of LATS1. European Review for Medical and Pharmacological Sciences, 21, 3402-3411.

[7] Wang, X.N., Zhang, L.H., Cui, X.D., et al. (2017) IncRNA HOXAll-AS Is Involved in Fracture Healing through Regulating mir-124-3p. European Review for Medical and Pharmacological Sciences, 21, 4771-4776.

[8] Jin, Q.S., Huang, L.J., Zhao, T.T., et al. (2018) HOXA11-AS Regulates Diabetic Arteriosclerosis-Related Inflammation via P131/AKT Pathway. European Review for Medical and Pharmacological Sciences, 22, 6912-6921.

[9] Nuermaimaiti, N., Liu, J., Liang, X.D., Jiao, Y., Zhang, D., Liu, L., et al. (2018) Effect of lncRNA HOXA11-AS 1 on Adipocyte Differentiation in Human Adipose-Derived Stem Cells. Biochemical and Biophysical Research Communications, 495, 18781884. https://doi.org/10.1016/j.bbrc.2017.12.006

[10] Skroblin, P. and Mayr, M. (2014) “Going Long”: Long Non-Coding RNAs as Biomarkers. Circulation Research, 115, 607-609. https://doi.org/10.1161/CIRCRESAHA.114.304839

[11] Wang, Q.X., Zhang, J.X., Liu, Y.W., Zhang, W., Zhou, J.H., Duan, R., et al. (2016) A Novel Cell Cycle-Associated lncRNA, HOXA11-AS, Is Transcribed from the 5-Prime End of the HOXA Transcript and Is a Biomarker of Progression in Glioma. Cancer Letters, 373, 251-259. https://doi.org/10.1016/j.canlet.2016.01.039 
[12] Zhang, Z.X., Zhou, J.H., Zhang, J.X., Duan, R., Pu, P.Y. and Han, L. (2017) Downregulation of lncRNA-HOXA11-AS Modulates Proliferation and Stemness in Glioma Cells. Chinese Neurosurgical Journal, 3, Article No. 25. https://doi.org/10.1186/s41016-017-0091-6

[13] Richards, E.J., Permuth-Wey, J., Li, Y.J., Chen, Y.A., Coppola, D., Reid, B.M., et al. (2015) A Functional Variant in HOXA11-AS, a Novel Long Non-Coding RNA, Inhibits the Oncogenic Phenotype of Epithelial Ovarian Cancer. Oncotarget, 6, 3474534757. https://doi.org/10.18632/oncotarget.5784

[14] Kim, H.J., Eoh, K.J., Kim, L.K., Nam, E.J., Yoon, S.O., Kim, K.-H., et al. (2016) The Long Noncoding RNA HOXA11 Antisense Induces Tumor Progression and Stemness Maintenance in Cervical Cancer. Oncotarget, 7, 83001-83016. https://doi.org/10.18632/oncotarget.12863

[15] Cui, M.X., Wang, J.Y., Li, Q.J., Zhang, J.L., Jia, J.L. and Zhan, X.L. (2017) Long Non-Coding RNA HOXA11-AS Functions as a Competing Endogenous RNA to Regulate ROCK1 Expression by Sponging miR-124-3p in Osteosarcoma. Biomedicine \& Pharmacotherapy, 92, 437-444. https://doi.org/10.1016/j.biopha.2017.05.081

[16] Zhang, H.C., Song, G.Y., Tan, W.J., et al. (2017) Effects of lncRNA HOXA11-AS on the Proliferation, Migration and Invasion of Osteosarcoma Cells. Chinese Journal of Cancer Biotherapy, 24, 497-502.

[17] Kogo, R., Shimamura, T., Mimori, K., Kawahara, K., Imoto, S., Sudo, T., et al. (2011) Long Noncoding RNA HOTAIR Regulates Polycomb-Dependent Chromatin Modification and Is Associated with Poor Prognosis in Colorectal Cancers. Cancer Reseach, 71, 6320-6326. https://doi.org/10.1158/0008-5472.CAN-11-1021

[18] Yim, G.W., Kim, H.J., Kim, L.K., Kim, S.W., Kim, S.H., Nam, E.J., et al. (2017) Long Non-Coding RNA HOXAI 1 Antisense Promotes Cell Proliferation and Invasion and Predicts Patient Prognosis in Serous Ovarian Cancer. Cancer Research and Treatment, 49, 656-668. https://doi.org/10.4143/crt.2016.263

[19] Zhang, Y., Chen, W.J., Gan, T.Q., Zhang, X.-L., Xie, Z.C., Ye, Z.H., et al. (2017) Clinical Significance and Effect of incRNA HOXA11-AS in NSCLC: A Study Based on Bioinformatics, in Vitro and in Vivo Verification. Scientific Reports, 7, Article No. 5567. https://doi.org/10.1038/s41598-017-05856-2

[20] Zhang, Y., He, R.Q., Dang, Y.W., Zhang, X.-L., Wang, X., Huang, S.-N., et al. (2016) Comprehensive Analysis of the Long Noncoding RNA HOXA11-AS Gene Interaction Regulatory Network in NSCLC Cells. Cancer Cell International, 16, Article No. 89. https://doi.org/10.1186/s12935-016-0366-6

[21] Sun, Y., Yang, J.X., Liu, B., Yang, B.Y. and Meng, Q. (2018) Long Non-Coding RNA Homeobox (HOX) A11-AS Promotes Malignant Progression of Glioma by Targeting miR-124-3p. Neoplasma, 65, 505-514. https://doi.org/10.4149/neo_2018 170705N462

[22] Zhang, Y., Luo, J., Wang, X., Wang, H.-L., Zhang, X.-L., Gan, T.-Q., et al. (2018) A Comprehensive Analysis of the Predicted Targets of miR-642b-3p Associated with the Long Non-Coding RNA HOXA11-AS in NSCLC Cells. Oncology Letters, 15, 6147-6160. https://doi.org/10.3892/ol.2018.8105

[23] Chen, J.H., Zhou, L.Y., Xu, S., Zheng, Y.-L., Wan, Y.-F., Hu, C.-P., et al. (2017) Overexpression of IncRNA HOXA11-AS Promotes Cell Epithelial-Mesenchymal Transition by Repressing miR-200b in Non-Small Cell Lung Cancer. Cancer Cell International, 17, Article No. 64. https://doi.org/10.1186/s12935-017-0433-7

[24] Yu, W., Peng, W., Jiang, H., Sha, H.Y. and Li, J.P. (2017) LncRNA HOXAI I-AS Promotes Proliferation and Invasion by Targeting miR-1 24 in Human Non-Small 
Cell Lung Cancer Cells. Tumor Biology, 39, 10104-10107. https://doi.org/10.1177/1010428317721440

[25] Zhao, X., Li, X.Y., Zhou, L.L., Ni, J., Yan, W.Y., Ma, R., et al. (2018) LncRNA HOXA11-AS Drives Cisplatin Resistance of Human LUAD Cells via Modulating miR-454-3p/Star3. Cancer Science, 109, 3068-3079. https://doi.org/10.1111/cas.13764

[26] Xue, J.Y., Huang, C., Wang, W., Li, H.B., Sun, M., Xie, M. (2018) HOXA11-AS: A Novel Regulator in Human Cancer Proliferation and Metastasis. OncoTargets and Therapy, 11, 4387-4393. https://doi.org/10.2147/OTT.S166961

[27] Sun, M, Nie, F., Wang, Y., Zhang, Z.H., Hou, J.K., He, D.D., et al. (2016) LncRNA HOXA 1 1-AS Promotes Proliferation and Invasion of Gastric Cancer by Scaffolding the Chromatin Modification Factors PRC2, LSDl, and DNMTI. Cancer Research, 76, 6299-6310. https://doi.org/10.1158/0008-5472.CAN-16-0356

[28] Wang, K.C. and Chang, H.Y. (2011) Molecular Mechanisms of Long Noncoding RNAs. Molecular Cell, 43, 904-914. https://doi.org/10.1016/j.molcel.2011.08.018

[29] Sun, L., Zhang, B.G., Liu, Y.Q., Shi, L.H., Li, H.L. and Lu, S.J. (2016) MiR125a-5p Acting as a Novel Gab2 Suppressor Inhibits invasion of Glioma. Molecular Carcinogenesis, 55, 40-51. https://doi.org/10.1002/mc.22256

[30] Orom, U.A., Derrien, T., Beringer, M., Gumireddy, K., Gardini, A., Bussotti, G., et al. (2010) Long Noncoding RNAs with Enhancer-Like Function in Human Cells. Cell, 143, 46-58. https://doi.org/10.1016/j.cell.2010.09.001

[31] Chen, D., Sun, Q., Cheng, X.F., Zhang, L.F., Song, W., Zhou, D.K., et al. (2016) Genome-Wide Analysis of Long Noncoding RNA (1ncRNA) Expression in Colorectal Cancer Tissues from Patients with Liver Metastasis. Cancer Medicine, 5, 1629-1639. https://doi.org/10.1002/cam4.738

[32] Li, T., Xu, C.F., Cai, B., Zhang, M., Gao, F. and Gan J.J. (2016) Expression and Clinicopathological Significance of the IncRNA HOXAI I-AS in Colorectal Cancer. Oncology Letters, 12, 4155-4160. https://doi.org/10.3892/ol.2016.5129

[33] Braconi, C., Kogure, T., Valeri, N., et al. (2011) microRNA-29 Can Regulate Expression of the Long Non-Coding RNA Gene MEG3 in Hepatocellular Cancer. Oncogene, 30, 4750-4756. https://doi.org/10.1038/onc.2011.193

[34] Chang, S., Liu, J.S., Guo, S.H., He, S.C., Qiu, G.L., Lu, J., Wang, J., Fan, L., Zhao, W. and Che, X.M. (2016) HOTTIP and HOXA13 Are Oncogenes Associated with Gastric Cancer Progression. Oncology Reports, 35, 3577-3785.

https://doi.org/10.3892/or.2016.4743

[35] Xu, C.Y., He, T., Li, Z.J., Liu, H.L. and Ding, B.Q. (2017) Regulation of HOXAI I-AS/miR-214-3p/EZH2 Axis on the Growth. Migration and Invasion of Glioma Cells. Biomedicine \& Pharmacotherapy, 95, 1504-1513.

https://doi.org/10.1016/j.biopha.2017.08.097

[36] Cui, Y., Yi, L., Zhao, J.Z. and Jiang, Y.-G. (2017) Long Noncoding RNA HOXA11AS Functions as miRNA Sponge to Promote the Glioma Tumorigenesis through Targeting miR-140-5p. DNA and Cell Biology, 36, 822-828. https://doi.org/10.1089/dna.2017.3805

[37] Sun, T.W., Wang, C.M., Xing, J. and Wu, D.Q. (2011) miR-429 Modulates the Expression of c-myc in Human Gastric Carcinoma Cells. European Journal of Cancer, 47, 2552-2559. https://doi.org/10.1016/j.ejca.2011.05.021

[38] Guo, J.-C., Yang, Y.-J., Zheng, J.-F., Zhang, J.-Q., Guo, M., Yang, X., et al. (2019) Silencing of Long Noncoding RNA HOXA11-AS Inhibits the Wnt Signaling Pathway via the Upregulation of HOXA11 and Thereby Inhibits the Proliferation, Inva- 
sion, and Self-Renewal of Hepatocellular Carcinoma Stem Cells. Experimental \& Molecular Medicine, 51, 1-20. https://doi.org/10.1038/s12276-019-0328-x

[39] Liu, B., Li, J., Liu, X.L., Zheng, M., Yang, Y., Lyu, Q., et al. (2017) Long Non-Coding RNA HOXA11-AS Promotes the Proliferation HCC Cells by Epigenetically Silencing DUSP5. Oncotarget, 8, 109509-109521. https://doi.org/10.18632/oncotarget.22723

[40] Ding, C., Cheng, S., Yang, Z., Lv, Z., Xiao, H., Du, C., Peng, C., Xie, H., Zhou, L., $\mathrm{Wu}$, J. and Zheng, S. (2014) Long Non-Coding RNA HOTAIR Promotes Cell Migration and Invasion via Down-Regulation of RNA Binding Motif Protein 38 in Hepatocellular Carcinoma Cells. International Journal of Molecular, 15, 4060-4076. https://doi.org/10.3390/ijms15034060

[41] Lu, Q.K., Zhao, N., Zha, G.P., Wang, H.Y., Tong, Q.H. and Xin, S.H. (2017) LncRNA HOXA11-AS Exerts Oncogenic Functions by Repressing p21 and miR-124 in Uveal Melanoma. DNA and Cell Biology, 36, 837-844. https://doi.org/10.1089/dna.2017.3808

[42] Steuer, C.E., El-Deiry, M., Parks, J.R., Higgins, K.A. and Saba, N.F. (2017) An Update on Larynx Cancer. CA: A Cancer Journal for Clinicians, 67, 31-50. https://doi.org/10.3322/caac.21386

[43] Qu, L., Jin, M., Yang, L., et al. (2018) Expression of Long Non-Coding RNA HOXA11-AS Is Correlated with Progression of Laryngeal Squamous Cell Carcinoma. American Journal of Translational Research, 10, 573-580.

[44] Sun, X.Y., Wang, X.F., Cui, Y.B., et al. (2018) Expression and Significance of Long Non-Coding RNA HOXA11-AS in Esophageal Squamous Cell Carcinoma. Chinese Journal of Oncology, 40, 186-190.

[45] Zhang, L., Wu, J. and Li, X.Y. (2018) Expression of Long Non-Coding RNAHOXAll-AS Affects the Migration of Head and Neck Squamous Cell Carcinoma Cells and the Prognosis of Patients. Journal of Xinxiang Medical College, 35, 310-313. 